\title{
Identification of Salmonella enterica serovar Kentucky genes involved in attachment to chicken skin
}

\author{
Sanaz Salehi ${ }^{1}$, Kevin Howe ${ }^{1}$, John Brooks ${ }^{2}$, Mark L. Lawrence ${ }^{3}$, R. Hartford Bailey ${ }^{1 *}$ and Attila Karsi ${ }^{3^{*}}$
}

\begin{abstract}
Background: Regardless of sanitation practices implemented to reduce Salmonella prevalence in poultry processing plants, the problem continues to be an issue. To gain an understanding of the attachment mechanism of Salmonella to broiler skin, a bioluminescent-based mutant screening assay was used. A random mutant library of a field-isolated bioluminescent strain of Salmonella enterica serovar Kentucky was constructed. Mutants' attachment to chicken skin was assessed in 96-well plates containing uniform $6 \mathrm{~mm}$ diameter pieces of circular chicken skin. After washing steps, mutants with reduced attachment were selected based on reduced bioluminescence, and transposon insertion sites were identified.
\end{abstract}

Results: Attachment attenuation was detected in transposon mutants with insertion in genes encoding flagella biosynthesis, lipopolysaccharide core biosynthesis protein, tryptophan biosynthesis, amino acid catabolism pathway, shikimate pathway, tricarboxylic acid (TCA) cycle, conjugative transfer system, multidrug resistant protein, and ATPbinding cassette (ABC) transporter system. In particular, mutations in S. Kentucky flagellar biosynthesis genes (flgA, flgC, flgK, flhB, and flg) led to the poorest attachment of the bacterium to skin.

Conclusions: The current study indicates that attachment of Salmonella to broiler skin is a multifactorial process, in which flagella play an important role.

Keywords: Salmonella, Kentucky, Flagella, Poultry, Skin, Attachment

\section{Background}

Salmonella contamination is an important food safety concern in poultry processing plants. Recently, Salmonella enterica serovar Kentucky has been recognized as the most prominent Salmonella serovar in poultry processing [1]. According to the National Antimicrobial Resistance Monitoring System (NARMS), the prevalence of $S$. Kentucky isolates from broiler chicken has elevated from $25 \%$ in 1997 to $50 \%$ in 2007 [2]. Although this serovar is not considered a major source of human disease, high incidence of Salmonella Kentucky and the emergence of its recent multi-drug resistant strain outside US with high resistance level to ciprofloxacin,

\footnotetext{
*Correspondence: rhbailey@cvm.msstate.edu; karsi@cvm.msstate.edu 'Department of Pathobiology and Population Medicine, College of Veterinary Medicine, Mississippi State University, Mississippi State, Mississippi, USA ${ }^{3}$ Department of Basic Sciences, College of Veterinary Medicine, Mississippi State University, Mississippi State, Mississippi, USA

Full list of author information is available at the end of the article
}

indicates this serovar could be a potential threat to public health.

Salmonella contamination persists in all stages of chicken processing regardless of the hygienic steps taken. While poultry intestines are considered the most probable origin of contamination, abundant bacteria have been detected on the surface of the broilers. There have been numerous studies on Salmonella attachment to chicken skin, however, specific knowledge on the mechanism of attachment is lacking. Bacterial attachment, according to one study, was a result of bacterial retention in a network of fibers that forms when chicken muscle fascia is immersed in water [3]. In another study, Salmonella isolation from cervices and feather follicles suggested that the bacterium can be entrapped in water inside the follicles [4]. While cell charge was considered an important attachment factor [5], another study indicated that cell charge did not affect the attachment rate [6]. Bacterial concentration and inoculation time are 
other aspects that have been suggested as contributors to the attachment of bacterium to chicken skin [7]. There also has been conflicting information on the role of some surface structures (e.g., fimbriae, pili, and flagella) on the attachment of the bacterium to the broiler skin surface [8-10].

Several Salmonella surface proteins that appear to mediate adhesion are involved into chicken fascia, which is composed of collagen and elastin fibers interspread in the glycosaminoglycan (GAG) matrix by binding hyaluronan [11]. However, the exact characteristics and properties of these binding sites are not completely understood.

The recent emergence of a ciprofloxacin-resistant strain in a broiler prevalent serovar of Salmonella [12] highlights the need to expand our knowledge of the $S$. Kentucky attachment mechanisms broiler skin. The purpose of this current research was to identify $S$. Kentucky genes mediating the bacterial attachment to chicken skin. Identification of Salmonella attachment mechanisms to poultry skin could allow development of strategies to reduce carcass contamination during processing, which could assist the broiler processing industry in meeting regulatory concerns for pre- and post-harvest food safety.

\section{Results}

\section{Identification of mutants with reduced skin attachment properties}

In total 2,112 S. Kentucky mutants were screened for their ability to bind to poultry skin. In the first screening, 264 candidate mutants with decreased bioluminescence relative to wild type $S$. Kentucky strain SkTn7lux were identified, including candidate mutants with decreased bioluminescence on chicken skin before and after the $1 \mathrm{~h}$ washing step (Fig. 1). Of these candidates, 88 showed a reduction in bioluminescence even before the washing step. The remaining 176 mutants had similar bioluminescence to strain SkTn7lux before washing but they displayed reduced bioluminescence after washing with agitation. After the second screening, we identified 66 mutants with decreased bioluminescence on chicken skin compared to strain SkTn7lux. Of these, 44 had decreased binding after the final wash step and 22 had decreased binding prior to the final wash. Wild type $S$. Kentucky was not removed from chicken skin after an hour of washing with agitation, while E. coli $\mathrm{DH} 5 \alpha$ was completely removed (Fig. 1). A total of 66 mutants showing complete or reduced attachment were chosen for transposon end mapping.

\section{Identification of transposon insertions in S. Kentucky genome}

Transposon insertion sites of 66 mutants with attenuated attachment to chicken skin were identified (Tables 1 and 2). Mutants that demonstrated attachment attenuation were classified into two phenotypic groups. The first group showed reduced skin attachment compared to $S$. Kentucky SkTn7lux only after $1 \mathrm{~h}$ washing with agitation. This group had transposon insertions in various genes: lipopolysaccharide (LPS) biosynthesis, amino acid catabolism, shikimate pathway, TCA cycle, conjugative transfer system (traD), signaling and transportation system, phage tail fiber protein $\mathrm{H}$, fimbrial export usher protein, membrane proteins, and several hypothetical proteins (Table 1). The second group had decreased skin attachment prior to $1 \mathrm{~h}$ washing. These mutants mostly had transposon insertions in different flagella structural genes (Table 2).

\section{Discussion}

In the current study, we used random transposon mutagenesis on bioluminescent Salmonella Kentucky strain SkTn7lux, to identify genes involved in attachment to chicken skin. Our results showed that $S$. Kentucky attachment to broiler skin is a multifactorial process requiring the expression of many genes. We identified two different phenotypic groups of mutants with decreased attachment on chicken skin. We expected to identify $S$. Kentucky mutants that were more susceptible to removal by washing, which mimics the washing process that occurs during the poultry processing. However, we also identified a group of mutants with more severe attachment defect, which had decreased skin binding after simple flushing with a pipette. Notably this second group included six mutants with transposon insertions in flagella genes highlighting the role of flagella in $S$. Kentucky attachment to poultry. These six mutants harbored two different transposon insertions in $f \lg A$, which encodes flagellar basal-body p-ring formation protein. Other mutated flagellar genes encode flagellar basalbody rod protein $(f l g C)$, flagellar hook-associated protein $(f l g K)$, a rod assembly protein $(f l g)$, and flagellar biosynthesis protein $(f l h B)$. Attachment defects in flagellar basal body protein mutants suggest that flagellar rotation contributes to skin attachment.

Previous studies have reported conflicting results on the role of flagella on attachment of Salmonella to broiler skin. In one study, attachment to broiler skin was dependent on the presence of flagella [10]. In later studies, it was concluded that under controlled conditions, non-flagellated bacteria attached as well as flagellated bacteria [9]. Similar to our results, attachment was found to be a complex reaction, and fimbria and flagella both contribute to the process [8].

Other attachment-defective mutations were in transporter and signaling systems, which have higher expression in attached bacteria, and may work as an efflux pump to help the bacterium resist environmental stress 

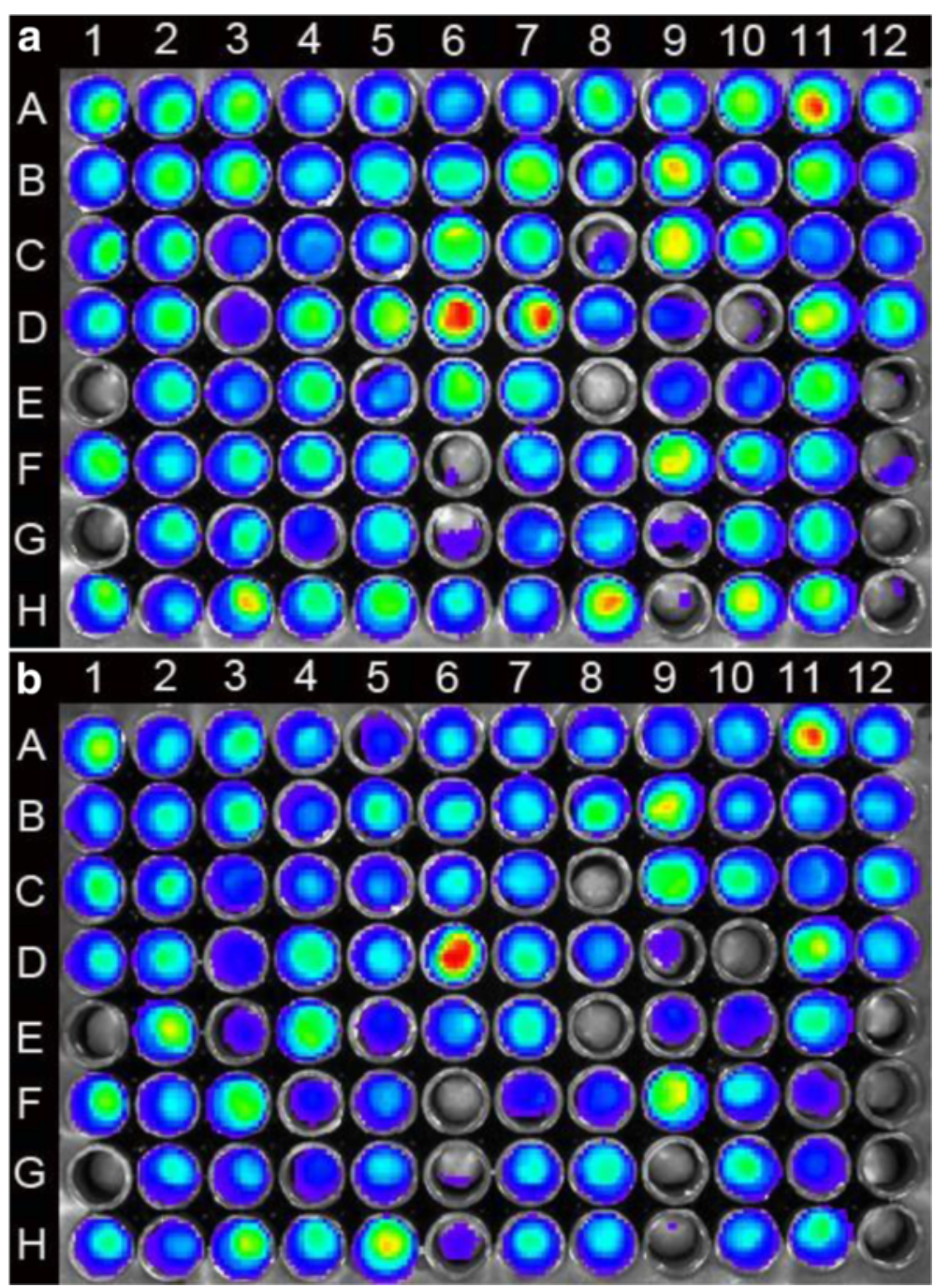

Fig. 1 Bioluminescence $\left(\mathrm{p} / \mathrm{s} / \mathrm{cm}^{2} / \mathrm{sr}\right)$ of 96-well plate containing chicken skin cuts: a before washing and $\mathbf{b}$ after washing of chicken skin cuts. The first four wells in the last column (A12, B12, C12, D12) are wild type S. Kentucky strain SkTn7lux and the last four wells in the same column (E12, $\mathrm{F} 12, \mathrm{G} 12, \mathrm{H} 12)$ are E. coli DH5a. The remaining wells are individual mutants that have been replicated in four plates. Examples of mutants with significantly decreased skin binding prior to the main wash are in wells E1, G1, F6, E8, D10 and H9

[13]. Thus mutation in these genes may make these mutants more susceptible to environmental conditions. In the current study, attachment-deficient mutant P07D05P01C06 had an insertion in lpp which encodes the major outer membrane lipoprotein. Under specific environmental conditions, an E.coli outer membrane lipoprotein, $\operatorname{NIp} E$, senses and generates an adhesion signal to the Cpx pathway that leads to stable adhesion [14]. Mutant P09H05-P01G06 had an insertion in the waaG gene, which encodes a LPS core biosynthesis protein. In E. coli, LPS is known to contribute to attachment. Some mutations in E. coli genes encoding lipopolysaccharide core biosynthesis enzymes showed decreased adhesion to solid surfaces [15]. In addition to waaG, two other mutants had insertions in LPS biosynthesis genes in the current study: P09B04-P01D09 was mutated in rffA which encodes a LPS biosynthesis protein; and P09E05P01F09 was mutated in rfaF, which encodes ADPheptose: LPS heptosyltransferase II that contributes to synthesis of the inner core backbone of LPS.

P25D03-P01C11 had an insertion in $\operatorname{trpB}$, which encodes tryptophan beta sub-unit synthase. Tryptophan is a major factor in forming Salmonella enterica serovar Typhimurium biofilms on food surfaces [16]. Amino acids metabolites, especially those involved in tryptophan biosynthesis are up-regulated at early stages of attachment. Although attachment of Salmonella to broiler skin is not considered a biofilm formation process, it is comparable with bacterial attachment to solid surfaces at an early stage of biofilm formation. In $E$. coli, over- 
Table 1 Skin attachment attenuated mutants removed after washing step

\begin{tabular}{|c|c|c|}
\hline Mutant $^{a}$ & Protein ID & Location $^{b}$ \\
\hline P02F10-P01G01 & 3-dehydroquinate dehydratase & MAR2XT7^TActgtccggtggttagcgcctgttcg \\
\hline P04G08-P01E02 & Magnesium and cobalt transport protein CorA & MAR2XT7^TAcgcgcaatcgctcgtcgtcgtccgg \\
\hline P07D05-P01C06 & Major outermembrane lipoprotein & MAR2XT7^TAaataccggaagtaatagttatcctg \\
\hline P07G06-P01E06 & Dihydrolipoamide acetyltransferase & MAR2xT7^TAtgtccgttcaccagaaacagcaaca \\
\hline P07G09-P01F06 & Dihydrolipoamide succinyl transferase & MAR2XT7^TAgctttcagtttcgcccgacgtatac \\
\hline P08F01-P01A08 & Poly nucleotide phosphorylase/polyadenylase & MAR2xT7^TAagcatggatgacaccgccgtattcg \\
\hline P08C05-P01B08 & Type IV conjugative transfer system coupling protein TraD & MAR2xT7^TAccaggaacgtcccaaagtggcgccg \\
\hline P09B04-P01D09 & Lipo polysaccharide biosynthesis protein RffA & MAR2xT7^TAtgtaacgtttaagcgcggcggtgtt \\
\hline P09E05-P01F09 & ADP-heptose:LPS heptosyl transferase ॥ & MAR2XT7^TAaacgaatttggcaacacccaggcgc \\
\hline P10H10-P01D10 & Anti-terminator-like protein & MAR2xT7^TAtattgataaacctcacgcccggcta \\
\hline P10D11-P01G11 & DNA helicase IV & MAR2XT7^TAtttgtcccgatcattcaaaacggcg \\
\hline P04H01-P01F02 & Phage tail fiber protein $\mathrm{H}$ & MAR2xT7^TActcacgtctggaaccaggttaccgg \\
\hline P06F05-P01H04 & Precorrin-4C11-methyl transferase & MAR2xT7^TAtgccggttcgctgatcaataccgaa \\
\hline P10F06-P01B11 & NADH pyro phosphatase & MAR2XT7^TAtggatcgtataattgaaaaattaga \\
\hline P10D07-P01G10 & Conserved protein with nucleoside triphosphate hydrolase Domain & MAR2xT7^TAgtgttcaagcagttgcaccatcgcg \\
\hline P08F09-P01H07 & Oligoribonuclease & MAR2XT7^TAtctaaacgcctttaccgatctgaaa \\
\hline P12F08-P02D02 & Glutamyl-Q tRNA (Asp) synthetase & MAR2XT7^TAtctccaccgccgcgacggactgttt \\
\hline P13H05-P02A03 & Chaperone protein HscA & MAR2XT7^TAtaccaactctctggttgcgacggtt \\
\hline P14B06-P02H03 & Chaperone protein HscA & MAR2XT7^TActgatcgtcgggcgcggcggcggtt \\
\hline P16D03-P02A05 & Shikimate 5-dehydrogenase AroDI gamma & MAR2XT7^TAcgaagcgctggatctcaattatctc \\
\hline P16H02-P02C05 & Fatty acid oxidation complex sub-unit alpha & MAR2XT7^TAcagcgggccgaggtgttgatactgc \\
\hline P17C05-P02A07 & SppA & MAR2XT7^TAatgctttatcctcaccaaggtacaa \\
\hline P18H08-P02C07 & NADH dehydrogenase sub-unit $\mathrm{H}$ & MAR2XT7^TAattgggtggtggccgatttaaacat \\
\hline P23E10-P02E10 & Ribulose-phosphate 3-epimerase & MAR2XT7^TAcactttgacgtcatggataatcact \\
\hline P25D03-P02C11 & Tryptophan synthase beta sub-unit & MAR2XT7^tgtgccgcagatcctgatgcctgcg \\
\hline P15C06-P02G04 & ATP-dependent RNA helicase DeaD & MAR2xT7^TAtaccgattgaagtgggecgtgatgt \\
\hline P11H11-P02B02 & Putative regulatory protein & MAR2xT7^TActgtcagcaatggccggaaaaagga \\
\hline P15C03-P02H04 & Glutathione reductase & MAR2xT7^TActtcatacgacaacgtgctgggcaa \\
\hline P21E02-P02C09 & Aldolase & MAR2XT7^TAtggtgtaatccagcaatttcctggc \\
\hline $\mathrm{P} 12 \mathrm{C} 04-\mathrm{P} 02 \mathrm{~F} 02$ & Putative sodium/sulfate transporter, partial & MAR2XT7^TAcagaatattggcggcggctttggct \\
\hline P18C07-P02F07 & GTP-binding protein & MAR2XT7^TActatcctcgctaaaaacaccgctat \\
\hline P23F01-P02D10 & Ornithine decarboxylase & MAR2XT7^TAgttggcctcttgcggattcatactg \\
\hline P16E01-P02B05 & Hypothetical protein STY0758 & MAR2XT7^TAccagggggactgacggcctgtgcag \\
\hline P19F07-P02H08 & Oxidoreductase & MAR2XT7^TAtattgagtcctcttccggcgtttcg \\
\hline P25G02-P02F11 & Intramembrane serine protease GlpG & MAR2xT7^TAtatatactgtattttgtatgga \\
\hline P19A07-P02A08 & Fimbrial outer membrane usher protein & MAR2XT7^TAcgttcggttcaatagcggtttcaat \\
\hline $\mathrm{P} 23 \mathrm{C} 06-\mathrm{P} 02 \mathrm{~B} 10$ & Pyruvate dehydrogenase sub-unit E1 & MAR2XT7^TAcatcaacactattgccgttgaagac \\
\hline P20C11-P02B09 & Alpha ribazole-5'-P phosphatase & MAR2xT7^TAcaaataatcatacagtcggacgata \\
\hline P18D02-P02G07 & 4-hydroxythreonine-4-phosphate dehydrogenase & MAR2xT7^TActctgctaggtgctgcccgacccgg \\
\hline P22G01-P02A10 & Permease protein SitC & MAR2xT7^TAagccatgcgcccagaaaactggtca \\
\hline Р13В03-P02Е03 & Putative sensor kinase protein & MAR2xT7^TAcaacaagaaatcgccgagcgcggac \\
\hline P10C09-P02C01 & Exoribonuclease II & MAR2xT7^TAtaaccagtcgccgacatcgcgctcc \\
\hline P22E10-P02G09 & Phosphorpyruvate hydratase & MAR2xT7^TAtcacaccaggcacagccgaccggac \\
\hline P19H03-P02B08 & High-affinity zinc transporter periplasmic protein & MAR2XT7^TAaaaccacgcgtacaagcgttgactt \\
\hline
\end{tabular}

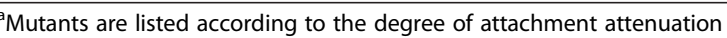

${ }^{\mathrm{b}}$ MAR2XT7, mariner transposon; $\wedge$, insertion point; TA, two-base TA duplication; lowercase letters, 25 -bp flanking unique gene sequences of $S$. enterica 
Table 2 Attachment attenuated mutants removed before the washing step

\begin{tabular}{|c|c|c|}
\hline Mutant $^{\mathrm{a}}$ & Protein ID & Location $^{\mathrm{b}}$ \\
\hline P09G05-P03F06 & Flagellar basal-body P-ring formation protein FlgA & MAR2XT7^TAttcatcgectgaccttccgcattga \\
\hline P03G04-P03G01 & Flagellar basal-body rod protein FlgC & MAR2xT7^TAgctgcgcaggctgacatcgtgttg \\
\hline P24B04-P03D10 & Unnamed protein product & MAR2xT7^TAttcccctggatgattttttacgcag \\
\hline P21C09-P03G09 & Flagellar biosynthesis protein FlhB & MAR2xT7^TAttccgtggcgctgcagtatgacgaa \\
\hline P05D08-P03D03 & Multidrug resistance protein, SMR family & MAR2xT7^TAcgcggcttaaaagggecaattcccg \\
\hline P05H05-P03C03 & Cysteine/glutathione ABC transporter membrane/ATP-binding comp. & MAR2XT7^TAgttaaaactgtaaattcccgcgaag \\
\hline P09H05-P03G06 & Lipopolysaccharide core biosynthesis protein & MAR2xT7^TAgcctgttctgggcgctgacagaaga \\
\hline P15B11-P03C08 & Flagellar hook-associated protein Flgk & MAR2XT7^TAgcaacagtaataatgccgataaaac \\
\hline P22D04-P03H09 & tRNAuridine5-carboxymethylaminomethyl modification enzyme GidA & MAR2xT7^TAacgaatcacgtcatgggttttctca \\
\hline P25E10-P03E10 & Flagellar rod assembly protein/muramidase FlgJ & MAR2XT7^TAcgttatagctgggttcgccattctc \\
\hline P16F02-P03F8 & DamX protein & MAR2xT7^TAtttgccgcacatgctgcgagataaa \\
\hline P13F05-P03G07 & NitrogenregulationproteinNR2, partial & MAR2XT7^TAcgtggcgcggcgcagctgcagagca \\
\hline P05E06-P03F02 & Dimethyl adenosine transferase & MAR2XT7^TAtttatcagcaggacgccatgaccat \\
\hline P17E05-P03D09 & 1-acyl-glycerol-3-phosphateacyltransferase & MAR2XT7^TAgaatgccgggctcttaggecttcag \\
\hline P12H05-P03F07 & Chain A, DNA-binding transcriptional repressor Acrr & MAR2xT7^TAagcaacgcgatggcgcgtaaaacca \\
\hline P25F11-PO3C11 & Cystathionine beta-lyase & MAR2XT7^TAtatgaccagccgcggtctgcgcaca \\
\hline P08C02-P03E05 & Flagellar basal body P-ring biosynthesis protein FlgA & MAR2xT7^TAttcatcgcctgaccttccgcattga \\
\hline P13C07-P03H07 & ParB gene product & MAR2xT7^TAcgactaaactcataagttaacgtac \\
\hline P02Е02-P03В01 & Two-component sensor kinase SsrA & MAR2xT7^TActtcgagtatggctggataaaacaa \\
\hline P16F04-P03G08 & Hemelyase sub-unit NrfE & MAR2xT7^TAtagcccgccagtaccacctgctgac \\
\hline P06D02-P03F04 & Hypothetical proteinSel_A3977 & MAR2xT7^TAaacactcaaaacgtcttggtattcg \\
\hline P05F11-P03H02 & Membrane protein suppressor for copper sensitivity ScsD & MAR2XT7^TAtaccgtgtcgggcgccggacattct \\
\hline
\end{tabular}

autants are listed according to the degree of attachment attenuation

${ }^{b}$ MAR2XT7, mariner transposon; $\wedge$, insertion point; TA, two-base TA duplication; lowercase letters, 25-bp flanking unique gene sequences of $S$. enterica

expression of tryptophan biosynthesis and increased production of tryptophan and its precursor, indole, prepares the bacteria for nutrient-poor environments and increases catabolism of amino acids. Indole also upregulates detoxifying genes (e.g., drug exporters) to make the bacterium more resistant to toxic compounds and increases bacterial adherence to surfaces [16]. These characteristics can be vital in Salmonella adherence to chicken skin. Also, P16D03-P01A05 and P02F10P01G01 had insertions in aroDI and aroD which encode 5-dehydrogenase gamma and 3-dehydroquinate dehydratase, respectively. These compounds are both enzymes in the Shikimate pathway and are involved in the biosynthesis of aromatic amino acids. These mutations also emphasize the importance of tryptophan in the attachment process.

\section{Conclusions}

Bioluminescence mutant screening of $S$. Kentucky was applied to identify mutants that are defective in attachment to chicken skin. Results indicate that flagella have an important role in attachment of $S$. Kentucky to broiler skin. Some other pathways that are important for skin adherence include LPS biosynthesis, aromatic amino acid biosynthesis, outer membrane lipoprotein, and transport/ secretion systems. Further investigations, especially in flagella structure and basal body genes, could lead to a better understanding of the exact molecular mechanism of Salmonella attachment to poultry skin.

\section{Methods}

Bacterial strains, plasmids and growth conditions

Escherichia coli SM10入pir [17] was used as the donor strain in conjugations for transfer of $\mathrm{p} M A R 2 x T 7$ [18] into bioluminescent $S$. Kentucky strain SkTn7lux [19] originally isolated from a broiler processing plant [20]. Bioluminescent E. coli DH5 $\alpha$ (Thermo Fisher Scientific, Waltham, MA) and bioluminescent wild type $S$. Kentucky strain SkTn7lux [19] were used as controls. E. coli and Salmonella strains were grown on Luria-Bertani (LB) broth and agar plates at $37{ }^{\circ} \mathrm{C}$. Gentamicin and streptomycin added to LB agar plates at $50 \mu \mathrm{g} / \mathrm{ml}^{-1}$ as appropriate.

\section{Construction of transposon insertion library}

pMAR2xT7 was transferred from E. coli SM10גpir into bioluminescent $S$. Kentucky strain SkTn7lux by conjugal 
mating [21]. Briefly, a colony of E. coli SM10גpir carrying $\mathrm{p} M A R 2 x T 7$ and bioluminescent $S$. Kentucky SkTn7lux were inoculated in $5 \mathrm{ml}$ of LB broth at $37{ }^{\circ} \mathrm{C}$ overnight at $200 \mathrm{rpm}$. Equal amounts of overnight culture of donor and recipient $(1.5 \mathrm{ml})$ were pelleted separately by centrifugation, washed three times with LB broth, and then re-suspended in $1 \mathrm{ml}$ of LB broth. Donor and recipient strains were mixed in a 1:3 ratio based on their volume. The mixture was centrifuged at $12,100 \mathrm{x} g$ for $2 \mathrm{~min}$. The harvested cells were diluted in $10 \mu \mathrm{l}$ of LB broth and transferred to a $0.45 \mu \mathrm{m}$ sterile filter paper, which was placed on LB agar and incubated at $37{ }^{\circ} \mathrm{C}$ for $18 \mathrm{~h}$. The filter was washed with $5 \mathrm{ml} \mathrm{LB}$ broth, and $50 \mu \mathrm{l}$ of the washed bacteria was spread on the LB agar containing gentamicin and streptomycin. Bioluminescence of colonies on agar plates were confirmed using an IVIS 100 Imaging System. A batch of gentamicin-resistant colonies was tested for random transposon insertion using single-primer PCR [22] and sequencing. More than 2,000 colonies of mutant S. Kentucky were picked using a pipette tip and inoculated in $150 \mu \mathrm{l}$ of LB plus gentamicin broth in 96-well plates and incubated in an incubator shaker overnight. Plates were sealed, and the mutant library was stored in $20 \%$ glycerol at $-80{ }^{\circ} \mathrm{C}[21]$.

\section{Chicken skin attachment assay}

In our previous studies, a chicken skin attachment assay was established, which indicated that the number of attached bacteria to chicken skin can be measured by the bioluminescence intensity of the correlated bacteria [23]. In this work, the chicken skin attachment assay was performed twice. Primary screening was accomplished with one replicate for a total of 2,112 mutants. Those mutants showing reduced attachment went through the final skin attachment assay with four replicates. Each 96-well plate contained four replicates of bioluminescent wild type $S$. Kentucky strain SkTn7lux and E. coli DH5a. Plates were covered with Breath-Easy film (Diversified Biotech, Boston, MA) and grown at $37{ }^{\circ} \mathrm{C}$ overnight at $250 \mathrm{rpm}$ on a shaker incubator. The OD and bioluminescence of each well were measured to ensure the growth and bioluminescence of each mutant. Five microliters from overnight cultures were used to inoculate fresh 96-well plate containing $100 \mu \mathrm{LB}$ broth, which were incubated at $37{ }^{\circ} \mathrm{C}$ for $2 \mathrm{~h}$ to reach the $\log$ phase.

The chicken skin was obtained from a commercial broiler processing plant inspected by USDA Food Safety Inspection Service. Chicken skins were cut into uniform, circular sections by $6 \mathrm{~mm}$ skin biopsy punch and placed into clear-bottomed 96-well black cell culture plates. $100 \mu \mathrm{l}$ of $\log$ phase mutant culture with known OD and bioluminescence were added to each well and after a brief spin, plates were incubated at $25^{\circ} \mathrm{C}$ for $1 \mathrm{~h}$ to allow bacterial attachment to the broiler skin. Following incubation, bacterial suspensions were removed by vacuum suction, and the wells were washed with $200 \mu$ of distilled water by pipetting twice to remove unattached bacteria. Plates were incubated at $37{ }^{\circ} \mathrm{C}$ for $10 \mathrm{~min}$. and bioluminescent imaging was recorded for $15 \mathrm{~s}$ of exposure at $37{ }^{\circ} \mathrm{C}$ with an IVIS 100 Imaging System. Bioluminescence was quantified using Living Images software as described [19].

To determine the effect of washing on bacterial attachment properties, plates were filled with $200 \mu \mathrm{l}$ of water and placed in a rotating platform incubator at $700 \mathrm{rpm}$ for $1 \mathrm{~h}$. After the removal of excess solution, bioluminescence on skin sections was measured and recorded for $15 \mathrm{~s}$ of exposure. This stage was considered as the main washing step.

\section{Determination of mutants with attenuated attachment}

Bioluminescence $\left(\mathrm{p} / \mathrm{s} / \mathrm{cm}^{2} / \mathrm{sr}\right)$ was measured twice in each assay: prior to and after the final $1 \mathrm{~h}$ washing step. In the primary screening, percent bioluminescence reduction was calculated from each mutant, which were then ranked from highest to lowest reduction. In the secondary screening, mutants with highest bioluminescence reduction went through another skin attachment assay with four replicates. Mutants were considered deficient in attachment if their attachment percentage fell out of the lower $95 \%$ confidence limit calculated from attachment rate of wild type strain SkTn7lux replicates. Mutants with decreased attachment either before or after the final wash were chosen for transposon end mapping.

\section{Identification of transposon insertion site}

Transposon insertion sites for the 66 mutants with reduced attachment to chicken skin were identified by overlap extension PCR [24]. Briefly, genomic DNA was prepared from overnight cultures using a Wizard Genomic DNA Purification Kit (Promega, Madison, WI). Each $25 \mu \mathrm{l}$ of PCR contained $0.2 \mu \mathrm{M}$ forward or reverse transposon specific primer (MAr2xT7F: TACAGTTT ACGAACCGAACAGGC or MAR2xT7R TCTATACAA AGTTGGGCATACGG) $0.2 \mathrm{mM}$ dNTPs, $1.5 \mathrm{mM} \mathrm{MgCl}$ and 1.25 U of Taq polymerase (Promega, Madison, WI). The PCR was performed using a PTC-100 thermocycler (MJ Research, Water town, MA) with the following cycling steps: initial denaturation $\left(2 \min\right.$ at $\left.94{ }^{\circ} \mathrm{C}\right)$ followed by 25 cycles of denaturation $\left(30 \mathrm{~s}\right.$ at $\left.94{ }^{\circ} \mathrm{C}\right)$, annealing $\left(30 \mathrm{~s}\right.$ at $\left.55^{\circ} \mathrm{C}\right)$, and elongation $\left(3 \mathrm{~min}\right.$ at $72{ }^{\circ} \mathrm{C}$ ) followed by 30 cycles of denaturation $\left(30 \mathrm{~s}\right.$ at $\left.94{ }^{\circ} \mathrm{C}\right)$, annealing $\left(30 \mathrm{~s}\right.$ at $\left.30{ }^{\circ} \mathrm{C}\right)$, and elongation $\left(2 \mathrm{~min}\right.$ at $\left.72{ }^{\circ} \mathrm{C}\right)$ followed by 30 cycle of denaturation $\left(30 \mathrm{~s}\right.$ at $\left.94^{\circ} \mathrm{C}\right)$, annealing $\left(30 \mathrm{~s}\right.$ at $\left.55^{\circ} \mathrm{C}\right)$, and elongation $\left(2 \mathrm{~min}\right.$ at $\left.72{ }^{\circ} \mathrm{C}\right)$. A final extension of $10 \mathrm{~min}$ at $72{ }^{\circ} \mathrm{C}$ was also applied. PCR 
products were cleaned with ExoSAP-IT enzyme mix (USB Corp. Cleveland, Ohio) and used as template in sequencing reactions using BigDye Terminator v1.1 and $0.5 \mu \mathrm{M}$ of a nested transposon specific primer (MAR2xT7FSeq: GGACCGAGATAGGGTTGAGTG or MAR2xT7R3Seq: AACAATTCGTTCAAGCCGAGA). Transposon specific sequences were trimmed and the remaining sequences were checked against the protein database of the National Center for Biotechnology Information (NCBI) using BLAST+, which revealed the location of transposon insertion [21].

\author{
Abbreviations \\ ABC, ATP-binding cassette; flg, flagellar; GAG, glycosaminoglycan; LPS, \\ lipopolysaccharide; NARMS, National Antimicrobial Resistance Monitoring \\ System; TCA, tricarboxylic acid.
}

\section{Acknowledgements}

We thank Dr. Scott Willard and Dr. Peter Ryan for use of the IVIS100 Imaging System in the Laboratory for Organismal and Cellular Imaging at the Department of Animal and Diary Sciences.

\section{Funding}

This project was funded by USDA ARS Agreement \#58-6402-2729, which is operated under USDA CRIS project MIS501170, "Mississippi Center for Food Safety and Post-Harvest Technology."

\section{Availability of data and material}

All data generated or analyzed during this study are included in this published article.

\section{Authors' contributions}

Conceived and designed the experiments: SS, KH, RHB, JB, MLL, AK. Performed the experiments: SS. Analyzed the data: SS. Contributed reagents/ materials/analysis tools: RHB, JB, MLL, AK. Wrote the paper: SS, KH, RHB, JB, $M L L, A K$. All authors have read and approved of the final version of the manuscript.

\section{Competing interests}

The authors declare that they have no competing interests.

\section{Consent for publication}

Not applicable.

\section{Ethics approval and consent to participate}

Not applicable.

\begin{abstract}
Author details
'Department of Pathobiology and Population Medicine, College of Veterinary Medicine, Mississippi State University, Mississippi State, Mississippi, USA. 2USDA-ARS, Genetics and Precision Agriculture Unit, Mississippi State, Mississippi, USA. ${ }^{3}$ Department of Basic Sciences, College of Veterinary Medicine, Mississippi State University, Mississippi State, Mississippi, USA.
\end{abstract}

Received: 22 January 2016 Accepted: 15 July 2016

Published online: 29 July 2016

\section{References}

1. Vasil ML. How we learnt about iron acquisition in Pseudomonas aeruginosa: a series of very fortunate events. Biometals. 2007:20(3-4):587-601.

2. Foley SL, Nayak R, Hanning IB, Johnson TJ, Han J, Ricke SC. Population dynamics of Salmonella enterica serotypes in commercial egg and poultry production. Appl Environ Microbiol. 2011;77(13):4273-9.

3. Thomas C, McMeekin T. Attachment of Salmonella spp. to chicken muscle surfaces. Appl Environ Microbiol. 1981:42(1):130-4.

4. Kim K, Frank J, Craven S. Three-dimensional visualization of Salmonella attachment to poultry skin using confocal scanning laser microscopy. Lett Appl Microbiol. 1996;22(4):280-2.
5. Dickson JS, Koohmaraie M. Cell surface charge characteristics and their relationship to bacterial attachment to meat surfaces. Appl Environ Microbiol. 1989;55(4):832-6.

6. Kim K, Lillard H, Frank J, Craven S. Attachment of Salmonella typhimurium to poultry skin as related to cell viability. J Food Sci. 1996;61 (2):439-41.

7. Kim K, Frank J, Craven S. Attachment of Salmonella on modified poultry skin surface. J Food Sci. 1996:61(2):442-4.

8. Lillard H. Role of fimbriae and flagella in the attachment of Salmonella typhimurium to poultry skin. J Food Sci. 1986;51(1):54-6.

9. Lillard H. Bacterial cell characteristics and conditions influencing their adhesion to poultry skin. J Food Prot. 1985:48:803-7.

10. Notermans $\mathrm{S}$, Kampelmacher E. Attachment of some bacterial strains to the skin of broiler chickens. Br Poultry Sci. 1974:15(6):573-85.

11. Sanderson $\mathrm{K}$, Thomas $\mathrm{C}$, McMeekin T. Molecular basis of the adhesion of Salmonella serotypes to chicken muscle fascia. Biofouling. 1991;5(1-2):89-101.

12. Le Hello S, Hendriksen RS, Doublet B, Fisher I, Nielsen EM, Whichard JM, Bouchrif B, Fashae K, Granier SA, Jourdan-Da Silva N. International spread of an epidemic population of Salmonella enterica serotype Kentucky ST198 resistant to ciprofloxacin. J Infect Dis. 2011;204(5):675-84.

13. Svensson S, Frirdich E, Gaynor E. Survival strategies of Campylobacter jejuni: stress responses, the viable but nonculturable state, and biofilms. In: Campylobacter. 3rd ed. Washington, DC: ASM Press; 2008. p. 571-90.

14. Otto K, Silhavy TJ. Surface sensing and adhesion of Escherichia coli controlled by the Cpx-signaling pathway. Proc Natl Acad Sci. 2002;99(4): 2287-92.

15. Genevaux P, Bauda P, DuBow MS, Oudega B. Identification of Tn10 insertions in the rfaG, rfaP, and galU genes involved in lipopolysaccharide core biosynthesis that affect Escherichia coli adhesion. Arch Microbiol. 1999; 172(1):1-8.

16. Hamilton S, Bongaerts R, Mulholland F, Cochrane B, Porter J, Lucchini S, Lappin-Scott H, Hinton J. The transcriptional programme of Salmonella enterica serovar Typhimurium reveals a key role for tryptophan metabolism in biofilms. BMC Genomics. 2009:10(1):599.

17. Miller VL, Mekalanos JJ. A novel suicide vector and its use in construction of insertion mutations: osmoregulation of outer membrane proteins and virulence determinants in Vibrio cholerae requires toxR. J Bacteriol. 1988; 170(6):2575-83.

18. Liberati NT, Urbach JM, Miyata S, Lee DG, Drenkard E, Wu G, Villanueva J, Wei T, Ausubel FM. An ordered, nonredundant library of Pseudomonas aeruginosa strain PA14 transposon insertion mutants. Proc Natl Acad Sci U S A. 2006;103(8):2833-8.

19. Howe K, Karsi A, Germon P, Wills RW, Lawrence ML, Bailey RH. Development of stable reporter system cloning luxCDABE genes into chromosome of Salmonella enterica serotypes using Tn7 transposon. BMC Microbiol. 2010; 10(1):197.

20. Volkova W, Bailey RH, Rybolt ML, Dazo-Galarneau K, Hubbard SA, Magee D, Byrd JA, Wills RW. Inter-relationships of Salmonella status of flock and growout environment at sequential segments in broiler production and processing. Zoonoses Public Health. 2010;57(7-8):463-75.

21. Karsi A, Gülsoy N, Corb E, Dumpala PR, Lawrence ML. High-throughput bioluminescence-based mutant screening strategy for identification of bacterial virulence genes. Appl Environ Microbiol. 2009;75(7):2166-75.

22. Karlyshev AV, Pallen MJ, Wren BW. Single-primer PCR procedure for rapid identification of transposon insertion sites. Biotechniques. 2000;28(6):1078. 1080, 1082

23. Karsi A, Howe K, Kirkpatrick TB, Wills RW, Bailey RH, Lawrence ML. Development of bioluminescent Salmonella strains for use in food safety. BMC Microbiol. 2008;8(1):10.

24. Horton RM, Hunt HD, Ho SN, Pullen JK, Pease LR. Engineering hybrid genes without the use of restriction enzymes: gene splicing by overlap extension. Gene. 1989:77(1):61-8. 